Ann. Génét. Sél. anim., I977, 9 (I), I7-26.

\title{
Mise au point sur le modèle classique d'estimation de la valeur génétique ${ }^{(1)}$
}

\author{
R. ROUVIER \\ Station d'Améliovation Génétique des Animaux, \\ Centre de Recherches de Toulouse, I.N.R.A., \\ $3_{3} 320$ Castanet Tolosan B.P. I2
}

\begin{abstract}
Résumé
Le modèle génétique et statistique classique d'estimation pour un ou plusieurs caractères, intra race ou souche, de la valeur génétique des reproducteurs, est présenté. C'est le modèle défini par Rouvier (1969a). Les travaux principaux antérieurs et postérieurs à cette date sont rappelés. Ce modèle consiste, dans l'hypothèse de distribution multinormale et donc de linéarité, à estimer les valeurs génétiques des caractères chez les candidats à la sélection par régression linéaire multiple sur les variables phénotypiques prédictrices. Cette estimation est l'index de sélection, sur un ou plusieurs caractères. Les paramètres nécessaires aux calculs sont obtenus à partir de l'expression de la covariance entre génotypes et phénotypes d'apparentés et par les méthodes statistiques usuelles. Quelques propriétés des index, lorsque les paramètres des populations sont supposés exactement connus, sont donnés. On a également discuté, dans le cas de la sélection par index sur plusieurs caractères, pour un génotype global, l'espérance du progrès génétique sur chaque caractère, ainsi que la sélection pour un objectif non linéaire.
\end{abstract}

L'objectif du sélectionneur est de changer les valeurs phénotypiques moyennes des populations animales étudiées, dans leur milieu de production, de façon à obtenir les phénotypes qui sont les plus désirables sur le plan économique ou sur celui de leur adaptation au milieu.

Seule la valeur génétique se transmettant de génération en génération, on est conduit à choisir les reproducteurs selon un prédicteur de celle-ci appelé couramment index ou indice de sélection.

Nous avons déjà développé l'étude du modèle statistique et génétique utilisé, de façon maintenant classique, pour estimer la valeur génétique des reproducteurs pour un ou plusieurs caractères (Rovvier, I $969 a$ ). Nous ferons donc ici un exposé de mise au point.

Nous nous limiterons au cas de la sélection sur les caractères à variation

(1) Cet article a fait l'objet d'une communication lors du séminaire sur " L'estimation de la valeur génétique des reproducteurs " organisé a Toulouse les 6 et 7 octobre 1976 par le Département de Génétique Animale de l'I.N.R.A. (France). 
continue, à déterminisme considéré comme polygénique, et à l'estimation de la valeur génétique par un index sur plusieurs caractères, le cas d'un seul devenant un cas particulier. Parmi les premiers travaux sur ce sujet, nous pouvons citer ceux de Sмith (I936) concernant une sélection sur descendance sur plusieurs caractères chez le blé; ceux de HAZEL (I943) dans le cas des animaux. Les premiers travaux sur l'indexation des animaux utilisent les cœfficients de piste de WRIGHT (I92I) pour calculer les corrélations entre phénotypes et génotypes d'individus apparentés. BOYER (I958) a de plus explicité l'idée importante suivant laquelle l'index de sélection s'écrit de façon simple comme étant l'équation de régression linéaire multiple de la valeur génotypique à estimer sur les variables phénotypiques prédictrices. La théorie des cœfficients de piste n'est pas d'utilisation généralisable dès que l'on veut considérer simultanément l'ensemble des parentés possibles, lorsque celles-ci sont de nombreux types, entre chaque individu et tous les autres de la population considérée. Il vaut alors mieux utiliser les formules générales : dérivée du modèle de CocKeRHAM (I954) dans le cas de populations panmictiques très grandes; de MATHERoN et al. (I974) dans le cas d'effets génétiques directs et maternels; de Gillois (I964), HARRIS (I964), dans le cas de populations consanguines.

Henderson (Ig63) a bien défini la théorie moderne de l'estimation de la valeur génétique des reproducteurs, à la fois sur le plan de l'expression de la covariance entre génotypes et phénotypes d'apparentés, et sur celui de l'établissement de l'index par régression linéaire multiple.

Depuis, l'utilisation de ce modèle s'est généralisée et certains développements ont été fait. Citons la synthèse récente de Gallais (r973). Citons aussi les développements relatifs à la sélection pour un objectif qui se présente comme une fonction non linéaire des valeurs génétiques de chaque caractère (Foulley et Rouvier, I97I), ainsi que la synthèse bibliographique critique de MALlaRD (I972) sur les index avec contraintes. Le modèle s'étend au cas où l'on considère les effets génétiques directs et maternels (VAN VLECK, I970). Ce modèle se prête bien, également, à la condensation de plusieurs index soit d'origines différentes (performance, ascendance, descendance) soit obtenus à des moments différents dans le temps sur le même animal (Colleau et Poutous, 1973) d'une part, et d'autre part la théorie de la sélection par étapes devient plus aisée à utiliser (RONNINGEN, I970).

Dans cette présentation, nous ne ferons pas une revue bibliographique, faite en r969. bien qu'il y ait eu, depuis, quelques travaux nouveaux surtout dans le domaine des applications. Nous présenterons, en résumé, ce qui semble être le modèle de base, maintenant classique du fait de son utilisation de plus en plus grande et efficace, de l'estimation de la valeur génétique des reproducteurs, pour un ou plusieurs caractères, à partir de toute l'information disponible (phénotypes de l'individu et de ses apparentés connus). Les démonstrations des résultats indiqués ont été faites précédemment (Rouvier, I969a et $b$, I970; Poujardieu et al., I974).

\section{I. - Modèle statistique d'estimation de la valeur génétique des reproducteurs Propriétés de l'index}

Le symbolisme matriciel utilisé avait déjà été introduit par Williams (I962a et $b$ ) au niveau d'un cas simple de sélection sur descendance et sur plusieurs caractères. 


\section{Soient :}

$g$ : le vecteur des valeurs génétiques des individus candidats à la sélection. Nous considérons une sélection intra race ou souche et, dans un premier temps, les valeurs génétiques additives des caractères. Si nous sélectionnon $q$ caractères, $g$ est un vecteur colonne à $q$ lignes.

$p$ : Le vecteur des variables aléatoires phénotypiques prédictrices. S'il y a $m$ prédicteurs, $p$ est un vecteur colonne à $m$ lignes. Les prédicteurs de la valeur génétique d'un individu peuvent être mesurés sur un seul ou sur plusieurs individus. Il peut $\mathrm{y}$ avoir des caractères prédicteurs différents de ceux dont on estime la valeur génétique.

Les individus considérés étant ceux d'une population, nous nous situons dans le cas d'un modèle à effets aléatoires et d'une distribution des vecteurs aléatoires $g$ et $p$. Nous ne considérerons pas ici, en prenant donc une hypothèse très simplificatrice, des effets fixés du milieu dont il faudrait tenir compte.

Constituons le tableau suivant, donnant les matrices de covariances des vecteurs $g$ et $p$ :

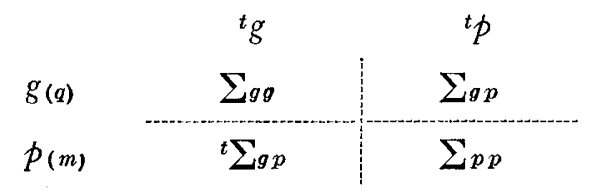

supposons les vecteurs $g$ et $p$ centrés. On a $\sum_{g g}=\mathrm{E}\left(g^{t} g\right)$. C'est la matrice de variance et covariance des valeurs génétiques additives à prédire.

$\sum_{g p}=\mathrm{E}\left(g^{t} p\right)$

$\sum_{p p}=\mathrm{E}\left(p^{t} p\right)$
Matrice de covariances des vecteurs $g$ et $p$

Matrice de variances et covariances des variables prédictrices.

Supposons une distribution multinormale du vecteur $\left(\frac{g}{p}\right)$ ce qui entraîne, notamment, la linéarité des liaisons.

La théorie de la régression linéaire multiple généralisée nous indique que l'estimation des $q$ valeurs génétiques additives des individus candidats à la sélection s'écrit :

$$
\mathrm{E}(g / p)=\widehat{g}=\sum_{g p} \sum_{p p}^{-1} p
$$

Cette estimation est la meilleure, linéaire, non biaisée et jouit de propriétés intéressantes dans l'hypothèse de distribution normale du vecteur $\left(\frac{g}{p}\right)$ et quand les 3 matrices sont exactement connues.

Lorsque la sélection est par index sur plusieurs caractères, le génotype global $\mathrm{H}$ (objectif) s'écrit :

$$
\mathrm{H}={ }^{t} a g
$$

$a$ étant un vecteur de cœfficients de pondération des valeurs génétiques additives des $q$ caractères. 
$H$ est estimé par I tel que la corrélation $R_{H x}$ entre $H$ et $I$ soit maximum. I est l'index de sélection sur plusieurs caractères. On peut montrer qu'il s'écrit:

$$
\mathrm{I}=\boldsymbol{t}_{a} \hat{\mathrm{g}}
$$

On peut démontrer que :

$$
\mathrm{R}_{\mathrm{HI}}^{2}=\frac{\sigma_{I}^{2}}{\sigma_{\mathrm{H}}^{2}}=\frac{{ }^{t} a \sum_{g p} \sum_{p p}^{-1} t \sum_{g p} a}{{ }_{t} a \sum_{g g} a}
$$

$\mathrm{R}_{\mathrm{HI}}^{2}$ mesure la précision de l'index. La variance $\sigma_{\mathrm{H}}^{2}$ étant fixée par la connaissance de la population et du vecteur $a$, on voit que la précision augmente avec $\sigma_{I}^{2}$ qui est la variance de la distribution des index. Lorsque la précision est plus grande, l'écart-type de la distribution des index est plus grand.

Un cas particulier d'estimation des valeurs génétiques des individus d'une population se présente lorsqu'il y a une information inégale pour estimer I. C'est le cas, par exemple, lorsque les tailles de famille sont déséquilibrées et donc lorsque le nombre d'individus apparentés est variable d'un candidat à la sélection à l'autre. Dans ces situations d'information inégale, les précisions des index se répartiront en différentes classes d'information et il y aura plusieurs distributions normales des index centrés caractérisées par leurs écarts types $\sigma_{\mathrm{r}}$. La question qui pouvait se poser était de savoir quelle troncature l'on doit utiliser sur la distribution de tous les index, pour sélectionner. HENDERson (I963) a montré qu'une troncature unique maximise la probabilité de discrimination dans le classement de deux individus, d'après la valeur de l'index. Nous avons montré que cette troncature unique maximise aussi l'espérance du progrès génétique. Cela à condition qu'il n'y ait pas de corrélation entre la moyenne génétique de l'index et sa précision. Ce cas pourrait se présenter: on peut imaginer, par exemple, que si l'on indexe une femelle (d'espèce polytoque) sur la prolificité de ses filles, il puisse y avoir une corrélation entre la valeur génétique de la femelle pour ce caractère, donc son index, et le nombre de filles, donc la précision de l'index. Dans les cas où il y aurait une telle liaison il faudrait faire une sélection par troncature sur la distribution des index centrés sur leur moyenne calculée dans chaque classe d'information de taille de famille. s'écrit :

L'espérance du progrès génétique, à la suite d'une sélection par troncature,

$$
\begin{aligned}
\mathrm{E}(\Delta \mathrm{H}) & =\frac{\operatorname{Cov}(\mathrm{H}, \mathrm{I})}{\sigma_{\mathrm{I}}^{2}} \Delta \mathrm{I} \\
& =\Delta \mathrm{I}
\end{aligned}
$$

$\Delta \mathrm{I}$ représente l'écart entre la moyenne du groupe sélectionné par troncature et la moyenne générale des individus indexés avant sélection. En effet $\operatorname{cov}(H, I)=\sigma_{I}^{2}$. Donc:

$$
\mathrm{E}(\Delta \mathrm{H})=i \sigma_{\mathrm{I}}
$$

$i$ étant l'intensité de sélection, c'est-à-dire, par définition,

$$
i=\frac{\Delta \mathrm{I}}{\sigma \mathrm{I}}
$$

On peut écrire aussi

$$
\mathrm{E}(\Delta \mathrm{H})=i \cdot \mathrm{R}_{\mathrm{HI}} \cdot \sigma_{\mathrm{H}}
$$


La sélection par index sur un seul caractère est un cas particulier de celle sur plusieurs caractères: soit $G$ la valeur génétique du caractère à estimer par un vecteur prédicteur $p$ supposé centré. On a:

$$
\widehat{\mathrm{G}}=(\operatorname{cov} \mathrm{G}, p) \sum_{p p}^{-1} p
$$

$\operatorname{cov}(\mathrm{G}, p)$ étant le vecteur des covariances entre $\mathrm{G}$ et le vecteur prédicteur $p$. tème

Si ${ }^{t} b p$ est la combinaison linéaire estimant $G$, il faut en effet résoudre le sys-

et

$$
\begin{aligned}
\sum_{p p} b & =\operatorname{Cov}(\mathrm{G}, p) \\
b & =\sum_{p p}^{-1} \operatorname{Cov}(\mathrm{G}, p)
\end{aligned}
$$

On a aussi

$$
\mathrm{R}_{\mathrm{GG}}^{2} \hat{\mathrm{G}}=\frac{{ }^{t} b \operatorname{cov}(\mathrm{G}, p)}{\sigma_{\mathrm{G}}^{2}}=\frac{{ }^{t} b \sum_{p p} b}{\sigma_{\mathrm{G}}^{2}}=\frac{\sigma_{\hat{\mathrm{G}}}^{2}}{\sigma_{\mathrm{G}}^{2}}
$$

$\widehat{G}$ étant l'index de sélection, l'espérance du progrès génétique suite à la sélection par troncature sur cet index s'écrit, avec

$$
\begin{aligned}
i & =\frac{\widehat{\Delta_{\mathrm{G}}}}{\sigma_{\mathrm{G}}} \\
\mathrm{E} \Delta \mathrm{G} & =i \cdot \hat{\sigma_{\mathrm{G}}} \\
& =i \cdot \mathrm{R}_{\mathrm{GG}} \cdot \sigma_{\mathrm{G}}
\end{aligned}
$$

Nous avons (PoujaRdieu et Rouvier, I972), à partir de ces formules d'expression de l'index pour un caractère, étudié l'optimum de la sélection combinée, telle qu'on peut la pratiquer en sélection des lapins, de la poule, des porcins, pour un troupeau contrôlé d'effectif donné (optimum de la répartition en familles de pères et mères).

\section{II. - Espérance du progrès génétique sur chaque caractère}

Que l'on sélectionne pour un seul ou pour plusieurs caractères à la fois, il est important d'exprimer le progrès génétique attendu sur chaque caractère, à la suite de cette sélection par troncature, sur une génération de sélection. Dans le cas de la sélection sur plusieurs caractères, Elston (I963) a défini un index indépendant des cœfficients de pondération. Indépendamment de ce cas particulier, le progrès attendu sur chaque caractère va dépendre notamment du vecteur $a$.

Ce vecteur $a$ peut-être défini de plusieurs façons; classiquement c'est le vecteur des cœefficients de pondération économique de chaque caractère. Cependant la valeur économique relative d'un caractère peut ne pas être constante ni dans l'espace ni dans le temps. La valeur économique d'un caractère peut ne pas toujours être proportionnelle au niveau génétique du caractère. Enfin, la valeur économique globale peut être une fonction non linéaire des valeurs génétiques de chaque caractère (MOAV et HILL, I966).

Le vecteur $a$ peut aussi être défini de façon à permettre d'atteindre un objectif. Un objectif réaliste est de faire évoluer les différents caractères dans des sens et suivant des rapports entre eux définis à l'avance. Cela se rapproche de l'objectif de sélection pour un idéal. Cela n'est qu'un cas particulier de la sélection par index avec contraintes (Mallard, I972). Rouvier, I969c, se plaçant d'un point de vue différent, avait proposé de déterminer $a$ de façon à maximiser $\sigma_{\mathrm{I}}$ ou $\sigma_{\mathrm{H}}$, ce 
qui permettrait de choisir les individus génétiquement extrêmes de la population et présenterait plutôt un intérêt expérimental.

Quel que soit le mode de détermination du vecteur $a$, il est important d'exprimer les progrès génétiques attendus sur chaque caractère à la suite de la sélection par troncature sur la valeur de l'index $\mathrm{I}$. Soit $\mathrm{E}(\Delta g)$ le vecteur qui les représente et $\mathrm{E}\left(\Delta_{1} \mathrm{G}\right), \mathrm{E}\left(\Delta_{2} \mathrm{G}\right), \ldots \mathrm{E}\left(\Delta_{q} \mathrm{G}\right)$, les progrès attendus sur chacun des caractères.

Il vient:

$$
\mathrm{E}(\Delta g)=\left[\begin{array}{l}
\mathrm{E}\left(\Delta_{1} \mathrm{G}\right) \\
\mathrm{E}\left(\Delta_{2} \mathrm{G}\right) \\
\cdot \\
\cdot \\
\cdot \\
\cdot \\
\cdot \\
\cdot \\
\mathrm{E}\left(\Delta_{q} \mathrm{G}\right)
\end{array}\right]=\frac{i}{\sigma_{\mathrm{I}}} \sum_{g p} \sum_{p p}^{-1} \sum_{g p} a
$$

Soient ${ }_{1} s,{ }_{2} s, \ldots q_{q} s$, les différentielles de sélection réalisées sur chaque caractère et $s$ le vecteur colonne correspondant de ces $q$ différentielles de sélection. On a

$$
\mathrm{E}(s)=\frac{i}{\sigma_{\mathrm{I}}}{ }^{t} a \sum_{g p}
$$

Dans chaque cas concret de sélection sur plusieurs caractères il sera intéressant de comparer les différentielles de sélection réalisées (vecteur $s$ ), leurs espérances (vecteur $\mathrm{E}(s)$ ) et les éléments du vecteur $\mathrm{E}(\Delta g)$. Poujardieu et al. (I974) ont montré l'intérêt concret d'une telle discussion dans un cas pratique de sélection sur descendance, sur 4 caractères, chez le lapin.

La formule ci-dessus permet inversement, sı l'on se donne $\mathrm{E}(\Delta g)$ comme objectif à une constante près de déterminer $a$ :

$$
=\frac{\sigma_{\mathrm{I}}}{i}\left(\sum_{g p} \sum_{p p}^{-1}{ }^{t} \sum_{g p}\right)^{-1} \mathrm{E} \Delta g
$$

La matrice qui intervient dans cette formule, sous forme de matrice inverse, est la matrice définie positive et symétrique, de variances et covariances des estimations ${ }_{i} \widehat{\mathrm{G}}$ de chaque ${ }_{i} \mathrm{G}$. En effet

$$
\mathrm{E}(\widehat{g} t \widehat{g})=\sum_{g p} \sum_{p p}^{-1}{ }^{t} \Sigma_{g p}
$$

Dans ce cas, dans lequel nous nous sommes placés, de distribution multinormale de l'ensemble des variables aléatoires considérées, l'équation d'un ellipsoide d'égale densité de probabilité des estimations $\hat{g}$ s'écrit:

$$
\widehat{t_{g}}\left(\sum_{g p} \sum_{p p}^{-1}{ }^{t} \sum_{g p}\right)^{-1} \widehat{g}=k
$$

En considérant que les valeurs du vecteur $\widehat{g}$ situées sur cet ellipsoïde, repérées par rapport à l'origine, sont telles que :

$$
\widehat{g}-o=\widehat{\Delta g}=\mathrm{E}(\Delta g)
$$


On obtient l'équation de l'ellipsoïde d'égale densité de probabilité des réponses.

$$
t[\mathrm{E}(\Delta g)]\left(\sum_{g p} \Sigma_{p p}^{-1}{ }^{t} \sum_{g p}\right)^{-1}[\mathrm{E}(\Delta g)]=k
$$

On peut montrer que la constante $k$ est égale à $i^{2}$, en remplaçant $\mathrm{E}(\Delta g)$ par son expression de la formule (I).

La considération de cet ellipsoïde des réponses (ou ellipse dans le cas particulier de deux caractères seulement) peut-être intéressante pour mieux juger de l'objectif de la sélection pratiquée par troncature sur l'index:

Mallard (exposé dans le cadre du présent séminaire) indique que l'on peut chercher, par tatonnement, un optimum empirique pour $\mathrm{E} \Delta g$.

MoAv et HrLl (r966) caractérisent, pour plusieurs espèces dans le cas de la production de viande, la valeur économique du couple mère-produits, ces produits étant destinés à la boucherie, par une fonction non linéaire des valeurs génétiques des aptitudes reproductives ${ }_{x} \mathrm{G}_{m}$ de la mère et productives ${ }_{y} \mathrm{G}_{p}$ des produits. SMITH (I964) dans sa comparaison de la sélection de lignées mixtes ou spécialisées paternelle et maternelle, considère comme critère une fonction linéaire de ${ }_{x} \mathrm{G}_{m}$ et de ${ }_{y} G_{p}$. Les résultats précédents permettent, dans le cas où la fonction économique $\mathrm{H}$ de deux caractères par exemple ${ }_{1} \mathrm{G}$ et ${ }_{2} \mathrm{G}$ n'est pas linéaire, de déterminer l'index linéaire I qui maximise le progrès attendu de cette fonction. Ainsi donc si l'objectıf est une fonction non linéaire $\operatorname{de}_{1} \widehat{\mathrm{G}}$ et ${ }_{2} \widehat{\mathrm{G}}$, on peut, en cherchant le point detangence de l'ellipse d'égale densité de probabilité de distribution de ${ }_{1} \widehat{\mathrm{G}}$ et ${ }_{2} \widehat{\mathrm{G}}$ et de la courbe représentative de cette fonction, déterminer le vecteur $\mathrm{E} \Delta g$ correspondant à ce point de tangence. C'est cette solution qui a été envisagée par MoAv et Hill. En déterminant alors le vecteur $a$ correspondant à $\mathrm{E} \Delta g$ ainsi choisi, on peut se ramener à un index linéaire.

\section{III. - Signification des matrices utilisées}

La connaissance de $\sum_{g g}$ suppose celle des variances et covariances génétiques des caractères.

Les éléments de $\sum_{g p}$ se calculent à partir de l'expression de la covariance entre génotypes d'une part, phénotypes d'autre part, d'individus apparentés. Le cas le plus simple est celui de population panmictique, infiniment nombreuse, en équilibre de linkage.

En écrivant que le phénotype est la somme des effets du génotype et du milieu

$$
\begin{gathered}
\mathrm{P}=\mathrm{G}+\mathrm{E} \\
\text { et } \operatorname{cov}(\mathrm{G}, \mathrm{P})=\operatorname{cov}(\mathrm{G}, \mathrm{G})+\operatorname{cov}(\mathrm{G}, \mathrm{E}) \quad \operatorname{avec} \operatorname{cov}(\mathrm{G}, \mathrm{E})=\mathrm{o}
\end{gathered}
$$

On a, pour deux individus $x$ et $y$ et pour le même caractère

$$
\operatorname{cov}\left(\mathrm{G}_{x}, \mathrm{P}_{y}\right)=\operatorname{cov}\left(\mathrm{G}_{x}, \mathrm{G}_{y}\right)=\sum_{1 \leqslant i+j \leqslant n}(2 \varphi)^{i} d^{j} \sigma_{i j}^{2}
$$

On utilise ici la formulation de Coc Kerham indiquée par CunNingham (I969) et Rouvier (I970). La covariance est due aux effets de gènes à $n$ loci. $\varphi$ est le cœfficient de parenté entre $x$ et $y$ défini par MALEcot (I948). $d$ est la probabilité d'identité des deux gènes situés à un locus, chez $x$ et $y . \sigma_{i j}^{2}$ est la variance génétique due à l'interaction de gènes seuls à $i$ loci et de paires de gènes à $j$ loci. La sommation a lieu sur tous les loci. $\sigma_{10}^{2}$ est donc la variance génétique additive; $\sigma_{01}^{2}$, celle de 
dominance; $\sigma_{20}^{2}$ la variance d'interaction additif $x$ additif; $\sigma_{11}^{2}$ la variance d'interaction additif $\times$ dominance; ...La formule se généralise au cas de la covariance entre génotype et phénotype, entre génotypes, pour deux caractères différents.

Le modèle génétique peut-être compliqué: effets génétiques liés au sexe; effets génétiques directs et maternels; populations consanguines, non panmictiques, sélectionnées. Des simplifications doivent en général être recherchées au niveau de l'estimation des composantes génétiques de la variance phénotypique.

Les éléments de $\sum_{p p}$ s'obtiennent à partir des méthodes statistiques usuelles, notamment d'estimation des composantes de la variance phénotypique ou de la régression entre phénotypes d'apparentés.

En pratique, on va donc égaler l'expression théorique des covariances entre apparentés aux covariances calculées entre phénotypes, de façon à estimer les différents éléments des matrices. Les méthodes statistiques usuelles (analyse de variance et covariance dans un modèle à effets aléatoires, régression) sont utilisables. Chevalet (1976) a récemment élaboré un modèle pour l'estimation des composantes de la variance phénotypique dans une population consanguine.

Williams J. S. (I $962 a, b)$ montrait déjà que le fait d'utiliser des paramètres estimés, dans une sélection par index sur descendance conduit à une valeur biaisée de $\mathrm{E}(\Delta g)$. Il s'agit en fait d'une source de variation du progrès génétique étudiée récemment par RAZUNGLEs (I974) dans le cas de la sélection sur un seul caractère.

\section{IV. - Conclusions}

Une grande clarté a pu être introduite dans le modèle d'estimation de la valeur génétique des reproducteurs, intra race ou souche. Il suffit de considérer que l'on cherche une estimation de la valeur génétique de chaque caractère par régression linéaire sur les variables phénotypiques prédictrices mesurables sur l'individu et ses apparentés. Sur le plan statistique l'on utilise la régression linéaire multiple et multivariable. Sur le plan génétique l'on utilise l'expression de la covariance entre génotypes et phénotypes d'individus apparentés. Lorsque la sélection porte sur plusieurs caractères et se fait par index sur plusieurs caractères, ceux-ci sont combinés linéairement après pondération ce qui donne le génotype global $H$ que l'on estime par l'index I, comme ci-dessus, tel que $R_{\mathrm{HI}}$ soit maximum. Dans le cas de distribution multinormale des variables génétiques et phénotypiques, et donc de linéarité, ces index sont les meilleurs estimateurs linéaires non biaisés.

Nous avons explicité ce modèle, donné les propriétés principales des index ainsi obtenus, et quelques limites. Ce modèle s'étend au cas de la sélection en croisement, que nous n'avons pas développée ici. L'élaboration de ce modèle classique a permis un certain nombre de développements, comme l'indiquent les exposés de ce séminaire du Département de Génétique animale.

\section{Notations}

Nous avons utilisé la notation matricielle suivante : une lettre capitale en traits gras représente une matrice, une lettre minuscule en italique un vecteur colonne, la lettre $t$ une transposition. Une lettre minuscule en italique précédée de $t$ représente donc un vecteur ligne. L'exposant $-\mathrm{I}$ signifie matrice inverse.

E signifie espérance mathématique et $\Delta$ accroissement. 


\title{
Summary
}

\author{
Classical model of estimation of genetic value : a review
}

The genetic and statistical model, from a classical point of view, for the estimation of breeding value for one or several traits at a time, within breed or strain, is presented. This model was already explained by Rouvier (1969a). Mains works on this subject are cited. This model permits, with hypothesis of multinormal distribution of genetic and phenotypic values, estimation of genetic values by multiple linear regression above phenotypic predictors. Necessary parameters for calculation are obtained from theoretical values of covariances between relatives and usual statistical methods. Some statistical properties of selection indexes, when parameters of populations are exactly known, are given. We discussed also, in the case of selection index for an aggregate genotype, the expected genetic improvement in each trait, and the selection for a non linear merit.

\section{Références bibliographiques}

Boyer J. P., 1958. Théorie et calcul des index de sélection. Ann. Zootech., 3, x93-242.

Colleau J. J., Poutous M., I973. Méthode de calcul des index de production laitière des femelles en France. Ann. Génét. Sél. anim., 5, 73-82.

Chevalet C., 1976. Estimation des composantes de la variance phénotypique dans une population consanguine. I. Élaboration du modèle. Ann. Génét. Sél. anim., 8, 181-206. - II. Application. Ann. Génét. Sél. anim., 8, 207-232.

Cockerham C. C., 1954. An extension of the concept of partitioning hereditary variance among relatives when epistasis is present. Genetics, 39, 859-882.

Cunningham E. P., 1969. Animal Breeding theory. Institute of Animal Genetics and Breeding, Landbruksbokhandefen. Universitets forlaget r969. Vollebekk, Oslo.

ELston R. C., I963. A weight free index for the purpose of ranking or selection with respect to several traits at a time. Biometrics, 19, 85-97.

Foulley J. L., Rouvier R., i97I. Méthodes d'établissement des index de sélection des taureaux de races à viande sur la valeur économique de leurs descendants. Ann. Génét. Sél. anim., 3, 497-507.

Gallais A., 1973. Sélection pour plusieurs caractères. Synthèse critique et généralisations. Ann. Amélior. Plantes, 23, I83-208.

Gillors M., I964. La relation d'identité en génétique. Thèse Fac. Sci. Paris.

HARRIS D. L., I964. Genotypic covariances between inbred relatives. Genetics, 50, I319-1348.

HAzEL L. N., 1943. The genetic basis for constructing selection indices. Genetics, 28,476-490.

Henderson C. R., I963. Selection index and expected genetic advance. Statistical genetics and plant breeding. Edited by W. D. Hanson and H. F. Robinson. Natl. Acad. Sci Nat. Res. Council Publ., 982, r4I-r63.

MALÉCOT G., I948. Les mathématiques de l'hévédité. Masson et Cie, Paris.

Mallard J., 1972. La théorie et le calcul des index de sélection avec restrictions : synthèse critique. Biometrics, 28, 7 $13-735$.

Matheron G., Poujardieu B., Lefort G., i974. Un modèle d'estimation des pavamètres génétiques en présence d'effets génétiques directs et maternels chez le lapin. I er Congrès Mondial de Génétique appliquée à l'Élevage. Madrid, 7-II octobre 1974.

Moav R., Hıll W. G., 1966. Specialised sire and dam lines. IV. Selection within lines. Anim. Prod., 8, 375-387.

Poujardieu B., Rouvier R., i97x. Optimisation du plan d'accouplement dans la sélection combinée. Ann. Génét. Sél. anim., 3, 509-5I9.

Poujardieu B.; Rouvier R., Vrillon J. L., Donal R., I974. Modèle de la sélection du lapin sur les caractères de croissance et d'efficacité alimentaire. I er Congrès Mondial de Génétique appliquée à l'Élevage. Madrid, 7-I I octobre 1974.

Razungles J., I974. Espérance et précision du progrès génétique. I. Précision de l'estimation des coefficients de régression dans l'index de sélection. Ann. Génét. Sél. anim., 6, 415-426. 
Rouvier R., I969a. Contribution à l'étude des index sur plusieurs caractères. Thèse $3^{\mathrm{e}}$ cycle, Fac. Sci. Paris.

Rouvier R., I969b. Calcul des index de sélection dans le cas d'individus consanguins et apparentés quelconques. Ann. Génét. Sél. anim., 1, 67-7o.

Rouvier R., I969c. Pondération des valeurs génotypiques dans la sélection par index sur plusieurs caractères. Biometrics, 25, 295-307.

Rouvier R., 1970. Génétique quantitative : $I^{\mathrm{re}}$ partie : Bases des méthodes et plans de sélection; $2^{\mathrm{e}}$ partie : la sélection pour l'élevage en croisement. Cours ronéotypé, Cours approfondi d'amélioration Génétique des animaux domestiques, I.N.A. Paris-Grignon.

RøNNINGEN K., I970. Studies on selection in animal breeding. II. The efficiency of selection based on the combination of performance testing and progeny testing compared with selection based only on performance testing when testing capacity is limited. Acta Agric. Scand., 20, $7 \mathrm{I}-\mathrm{IO} 4$.

Smith C., 1964. The use of specialised sire and dam lines in selection for meat production. Anim. Prod., 6, 337-344.

Smith H. F., 1936. A discriminant function for plant selection. Annals Eugenics, 7, $240-25^{\circ}$.

VAN VLECK I. D., I970. Index selection for direct and maternal genetic components of economic importance. Biometrics, 26, 477-483.

Williams J. S., rg62a. The evaluation of a selection index. Biometrics, 18, 375-393.

Williams J. S., r962b. Some statistical properties of a genetic selection index. Biometrika, 49, 325-337.

Wright S., I92I. Systems of mating. I. The biometric relations between parent and offspring. Genetics, 6, II I-I 23 . 